\title{
Transfer of associative grouping to novel perceptual contexts in infancy
}

\author{
Ashley Kangas • Nicole Zieber • Angela Hayden • \\ Paul C. Quinn • Ramesh S. Bhatt
}

Published online: 9 August 2011

(C) Psychonomic Society, Inc. 2011

\begin{abstract}
Learning can be highly adaptive if associations learned in one context are generalized to novel contexts. We examined the development of such generalization in infancy in the context of grouping. In Experiment 1, 3- to 4-month-olds and 6- to 7-month-olds were habituated to shapes grouped via the organizational principle of common region and were tested with familiar and novel pairs as determined by the principle of proximity. Older infants generalized from common region to proximity, but younger infants did not. Younger infants failed to generalize when the task was easier (Experiment 2), and their failure was not due to inability to group via proximity (Experiment 3). However, in Experiment 4, even younger infants generalized grouping on the basis of connectedness to proximity. Thus, the ability to transfer learned associations of shapes to novel contexts is evident early in life, although it continues to undergo quantitative change during infancy. Moreover, the operation of this generalization mechanism may be induced by means of bootstrapping onto functional organizational principles, which is consistent with a developmental framework in which core processes scaffold learning.
\end{abstract}

Keywords Infant development · Perceptual learning . Perceptual organization - Gestalt grouping

\footnotetext{
A. Kangas $\cdot$ N. Zieber $\cdot$ A. Hayden $\cdot$ R. S. Bhatt $(\square)$ Department of Psychology, University of Kentucky, Lexington, KY 40506-0044, USA

e-mail: rbhatt@email.uky.edu

P. C. Quinn

Department of Psychology, University of Delaware, Newark, USA
}

The sensory information that we receive about objects in our environment can be incomplete and have multiple interpretations. However, we experience the world in a meaningful way because our visual system is able to organize this information into coherent percepts (Palmer, 1999). Gestalt psychologists have suggested that this organization is governed by rules that are based on the specific structure of the human brain and are not individually learned (Koffka, 1935; Kohler, 1929; Wertheimer, 1923/1958). Research indicates, however, that learning plays a powerful role in the manner in which we organize information (e.g., Gibson, 1969; Goldstone, 1998, 2003; Peterson \& Salvagio, 2008). For example, learning has been shown to affect fundamental perceptual organizational processes such as figure-ground segregation: Prior knowledge of objects' silhouettes can determine which portion of an image is deemed the figure (e.g., Peterson, 1994).

Learning has also been shown to be a major contributor to perceptual organization in infancy (Bhatt \& Quinn, 2011; Johnson, 2010). For instance, Johnson (2009) suggested that learning enables infants to perceptually complete images of objects that are partly occluded. Baillargeon, $\mathrm{Li}, \mathrm{Ng}$, and Yuan (2009) similarly concluded that infants' understanding of physical events is facilitated by learning about the roles of different variables that may impact the events.

Learning can be adaptive by allowing the formation of associations that are useful in a particular context. However, learning can be even more powerful if learned associations are carried forward into new contexts. The learned associations may enable the perceptual structuring of the scene in the new context. Vickery and Jiang (2009) provided evidence for this idea: Adult participants in their study transferred learned associations of shapes to a new context, and the organization of the scene in the new 
context was driven by the previously learned association. Similarly, generalization of learning to new situations is fundamental to a number of significant perceptual and cognitive functions, such as categorization (Murphy, 2002).

The goal of the present research was to examine infants' generalization of learned associations to new contexts. Specifically, we examined whether infants' recognition of the structure of novel scenes is affected by previously learned associations. If so, it would indicate that past experience transfers to new contexts and can thus be a powerful determinant of perceptual organization in infancy.

There is ample evidence that infants are sensitive to association between features in particular contexts. For example, Slater, Mattock, Brown, Burnham, and Young (1991) found that newborns are sensitive to the correlations between the color and orientation of lines. Similarly, Bhatt and Rovee-Collier $(1994,1996)$ found that 3-month-olds are sensitive to the correlation between colors and shapes displayed on mobile blocks. Several statistical learning studies have demonstrated that infants are sensitive to spatiotemporal correlations in visual and auditory domains (e.g., Fiser \& Aslin, 2002; Kirkham, Slemmer, \& Johnson, 2002; Saffran, 2009). Furthermore, Feigenson and Halberda (2008) found that providing appropriate spatiotemporal information allows infants to register the correlation between objects and chunk them into object arrays, resulting in increased memory capacity. While these studies indicate that infants are sensitive to correlations based on spatiotemporal information, we asked whether infants' transfer previously learned associations to novel contexts.

The present research also addressed developmental changes in the transfer of learned associations. Some prior reports indicated developmental changes in infants' sensitivity to correlations among features. For instance, Younger and Cohen (1986; see also Cohen, 1991, 2010) suggested that young infants initially perceive individual features only and that the ability to perceive relations among features develops later in development. The fact that even newborns process correlations among features under certain conditions (Slater, Mattock, Brown, Burnham, \& Young, 1991) argues against the strong view that young infants are unable to process correlations; yet it is possible that the degree to which correlations are processed might change with development. Similarly, there may be developmental changes in generalization of associations. That is, under certain conditions, older infants' might transfer learned associations to new scenes, but younger infants might not. To examine this issue, we tested both 3- to 4-month-old and 6- to 7-month-old infants in the present experiments. We chose these age groups because Cohen and his colleagues have documented developmental changes in the processing of feature correlations across these ages (Cohen, 2010; Younger \& Cohen, 1986) and because other studies have revealed developmental changes in global processing of faces and nonface patterns from 3 to 6 months of age (e.g., Bhatt, Bertin, Hayden, \& Reed, 2005; Cashon \& Cohen, 2004; Quinn, Bhatt, Brush, Grimes, \& Sharpnack, 2002).

\section{Experiment 1}

Prior research indicates that 3- to 4-month-olds and 6- to 7-month-olds group shapes on the basis of the Gestalt principle of common region (Bhatt, Hayden, \& Quinn, 2007; Hayden, Bhatt, \& Quinn, 2008). The common region principle states that, all else being equal, elements that are within a region are grouped together and separated from those in other regions (Palmer, 1992). In Bhatt, Hayden, and Quinn (2007), infants were familiarized with two pairs of shapes; one pair (e.g., A and B) was located together in one region, while the other pair (e.g., C and D) was located together in another region. Infants were then tested with a familiar within-region grouping (e.g., A with B) and a novel between-region grouping (e.g., B with $\mathrm{C}$ ). The infants discriminated between the grouping of elements that had shared a common region during habituation and the grouping of elements from different regions. These results indicate that infants grouped element shapes in accord with the principle of common region (Palmer, 1992). Infants are also sensitive to the principle of proximity, as demonstrated by 3- to 4-month-olds' utilization of spatial proximity relations to organize patterns of elements into holistic columns and rows (Quinn, Bhatt, \& Hayden, 2008). Thus, prior research indicates that infants as young as 3 to 4 months of age organize visual patterns on the basis of both common region and proximity. In Experiment 1, we utilized infants' sensitivity to common region and proximity to examine transfer of learning. If infants transfer grouping based on common region to organization based on proximity relations, then it would suggest that learned associations transfer across contexts in infancy and affect the recognition of the structure of novel scenes.

\section{Method}

Participants Twenty 6- to 7-month-old infants (14 males; mean age $=204.4$ days, $S D=10.4)$ and twenty 3 - to 4-month-olds (10 males; mean age $=106.5$ days, $S D=8.8$ ) participated in the present experiment. Data from an additional older infant and 6 younger infants were excluded due to failure to sample both test stimuli (greater than $90 \%$ looking toward one pattern; $n=4)$, fussiness $(n=2)$, or disinterest (looking less than $1 \mathrm{~s}$ during test; $n=1$ ). Infants were recruited through local birth announcements and from a local hospital. They were predominantly Caucasians from middle class families. 
Stimuli The stimuli used during habituation were the same as those used in Bhatt et al. (2007). On each trial, identical habituation patterns were presented on both sides of the monitor. Each infant was habituated to two pairs of elements that were located in different vertical or horizontal regions (Fig. 1). The top-bottom and left-right distances between the elements were equated such that the pairings could be defined only by the presence of the elements within regions, and not by proximity relations. To ensure that particular elements were not associated with particular spatial positions or regions, the left-right or top-bottom positions of the elements and the location of the pairs in regions were varied across trials in a counterbalanced manner, although each pair of grouped elements always shared a region (Fig. 1). Thus, the pairings were defined by the fact that elements of a group always shared a region, although this region varied from one trial to the next. Each element subtended approximately $1.43^{\circ}$, and the horizontal/ vertical space between them subtended $3.42^{\circ}$.

Two different two-pair combinations were derived from the shapes $\mathrm{H}, \mathrm{X}, \mathrm{O}$, and square. Half of the infants were habituated to one combination, while the other half was habituated to the other set. The combinations were such that each pair of elements appeared equally often as a withinregion and a between-region pair during the test.

After habituation, infants were tested for preference between a familiar versus a novel grouping of element pairs, where the groupings were determined by proximity (Fig. 1). The within-region and between-region pairs of test stimuli were counterbalanced across infants, such that a pair that had shared a common region during habituation for half of the infants was the pair that came from different regions for the other half. Thus, any preference during the test could be due only to the status of the pairs during habituation, rather than to a priori preferences. During the tests, the distance between the elements within a pair was $0.80^{\circ}$, while the distance between the pairs was $3.42^{\circ}$. At test, the element pairs were oriented differently than during habituation. That is, if grouping was based on vertical regions during habituation, the grouping that defined novelty/familiarity during the test would be based on horizontal proximity relations, and vice versa (Fig. 1). This change was intended as a further test of infants' abilities to generalize grouping to a novel situation (Bhatt et al., 2007; Hayden et al., 2008). Also, for half of the infants, the familiar-grouped stimuli (i.e., elements that had been located in a common region during habituation) were presented on the left side of the computer monitor on the first test trial, while the novel-grouped stimuli (i.e., elements that had been located in different regions during habituation) were presented on the right. For the other half of the infants, these positions were reversed. Also, the stimulus positions were reversed from the first to the second test trial for all infants.

\section{Habituation}

Trial 1
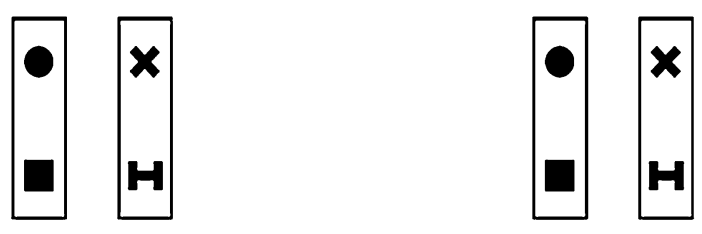

Trial 2
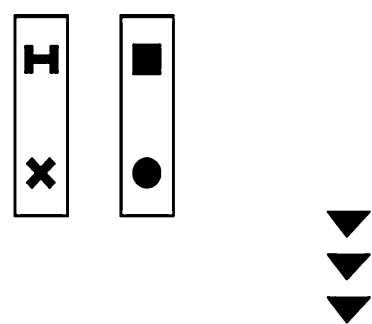

2

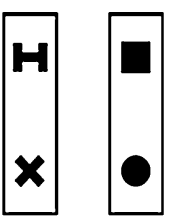

Familiar Grouping

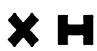

Novel Grouping
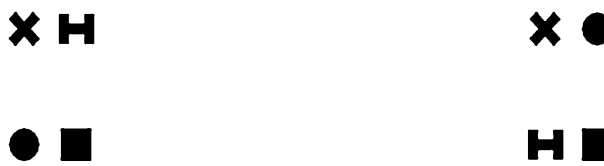

Fig. 1 Examples of the stimuli used in Experiment 1. Infants were habituated to two pairs of shapes contained within regions, with one pair sharing a horizontal or vertical region and the other a different horizontal or vertical region. Infants were then tested for their preference between pairs of shapes that had shared regions during habituation versus pairs of shapes that had been in different regions during habituation, with the pairings during the test defined by proximity relations. There was an orientation change from habituation to test, such that infants habituated to grouping based on vertical regions during habituation were tested with pairings based on horizontal proximity relations and vice versa. The pairs of shapes that made up the within-region and between-region patterns during habituation were counterbalanced across infants; thus, within each age group, each pair of elements equally often reflected familiar and novel pairing during the test

Apparatus and procedure Infants were seated approximately $45 \mathrm{~cm}$ from a $50-\mathrm{cm}$ computer monitor in a darkened chamber. A video camera (mounted on top of the monitor) and a DVD recorder (located outside of the test chamber) were used to record infants' looks. The infant-control habituation procedure used in this study was the same as that used in previous studies that have found grouping based on common region in infancy (Bhatt et al., 2007; Hayden et al., 2008; see Horowitz, Paden, Bhana, \& Self, 1972). Each trial began with a colorful attention getter (pink/green alternating shapes) located centrally on the monitor. Once the infant 
fixated this spot, the experimenter pressed a button to start the trial. Two identical habituation stimuli appeared on the screen, located to the left and right of center. The stimuli remained on the screen until infants looked away for $2 \mathrm{~s}$ or until a maximum of $60 \mathrm{~s}$ had elapsed. As described above, the locations of the stimulus elements varied across trials, although the elements of a pair always shared the same region (Fig. 1). Trials continued in this manner until the mean look duration on 3 consecutive trials was less than $50 \%$ of the average look duration during the first 3 habituation trials or until a maximum of 20 trials was reached.

Two 10-s test trials followed habituation. Each trial started with the fixation of the infants' gaze on the center of the screen induced by the attention getter. Thereafter, the test stimuli remained on the screen for $10 \mathrm{~s}$. That is, the duration of the test trials was independent of the infant's behavior. During each test trial, a novel grouping of elements was presented on one side of the monitor, and a familiar grouping was presented on the other side, with the groupings based on proximity (Fig. 1). Infants' behavior during the tests was coded offline by an observer who was not aware of the left/right position of the test images. Videos were slowed to $25 \%$ speed during coding of test performance. Coding reliability was assessed by a second coder on a randomly selected subset of 10 (5 older, 5 younger) infants. The Pearson correlation coefficient between the two coders' scores was .93 . The reliability of measurement during habituation was also assessed by a second coder (offline from video), and the Pearson correlation coefficient between the two coders was .94.

\section{Results and discussion}

Except for 2 older infants, all infants habituated within the 20 -trial limit. The pattern of results did not differ whether the data from the 2 participants were included or not. In the following analyses, the data from all the participants are included. Mean number of trials to habituate and infants' look durations during the first three and last three habituation trials are shown in Table 1. A $t$-test failed to reveal a difference between the age groups in terms of number of trials to habituate, $t(38)=1.32, p>.10$. Moreover, an analysis of infants' look durations during the first three and last three habituation trials, using an age (3- to 4-month-olds, 6- to 7-month-olds) $\times$ trials (first three, last three) ANOVA, revealed a significant main effect of trials, $F(1,38)=73.64, p<.001, \eta_{\mathrm{p}}{ }^{2}=.66$. Neither age nor the interaction was significant. Thus, as required by the procedure, infants' look durations declined significantly from the first three to the last three trials, and infants in both age groups were habituated to a similar extent before being tested.
As in previous studies (e.g., Bhatt et al., 2007; Hayden et al., 2008; Quinn et al., 2002), infants' performance during the test was assessed by calculating a mean novelty preference score. This score was calculated by dividing the look duration to the novel grouping by the total duration of looking toward both the novel and familiar groupings and then multiplying this ratio by 100 to obtain a percent preference score. The mean novelty preference score was then compared with the chance level of $50 \%$ to assess infants' performance. As in previous studies, a novelty preference score that was significantly greater than $50 \%$ was taken to indicate discrimination of the novel grouping of stimuli from the familiar grouping.

Older infants generalized groupings, but younger infants did not (Table 1). Six- to 7-month-olds exhibited a mean preference for the novel grouping of shapes that was significantly different from the chance level of $50 \%, t$ $(19)=2.82, p<.02$. However, 3 - to 4-month-olds failed to exhibit a preference, $t(19)=0.97, p>.3$. Furthermore, an independent samples $t$-test indicated a reliable difference between the older and younger infants' mean preference scores, $t(38)=2.29, p<.05, d=0.72$. These results suggest that 6- to 7-month-olds learned associations as revealed by their generalization of grouping based on common region to grouping based on proximity. However, 3- to 4-month-olds failed to exhibit evidence of generalization.

One issue to consider is the fact that the older infants exhibited a preference for novel organization during the tests in the present experiment, while infants in Bhatt et al. (2007) exhibited a familiarity preference when tested with shape combinations organized on the basis of common region during both habituation and test. Several factors affect whether infants exhibit a novelty or familiarity preference, including the age of the infants, the degree of habituation, and the complexity of the stimuli (Bhatt, Hayden, Reed, Bertin, \& Joseph, 2006; Cohen, 2004; Fiser \& Aslin, 2002; Hunter \& Ames, 1988; Roder, Bushnell, \& Sasseville, 2000). The difference between the Bhatt et al. (2007) experiments and Experiment 1 is the use of common region versus proximity as the organizing cue during the test. It is possible that organization via proximity is less complex and more robust than organization via common region, and this may have led to novelty preference in Experiment 1 and familiarity preference in Bhatt et al. (2007), because increased complexity is thought to lead to familiarity preference. However, in Experiment 1, there was a change in organizational cues from habituation to test, whereas there was no change in Bhatt et al. (2007); thus, one could argue that complexity was higher in Experiment 1. It is thus unclear as to why older infants exhibited a novelty preference in Experiment 1, while same-age infants exhibited a familiarity preference in Bhatt et al. (2007). 
Table 1 Means (and standard errors) for number of trials to habituate, fixation duration during habituation trials, and percent preference for the novel pairing of elements exhibited during test trials

\begin{tabular}{|c|c|c|c|c|c|c|}
\hline & \multicolumn{4}{|c|}{ HABITUATION } & \multirow{2}{*}{\multicolumn{2}{|c|}{$\begin{array}{l}\text { TEST } \\
\text { Preference for Novel Pairing (\%) }\end{array}$}} \\
\hline & \multirow[t]{2}{*}{$N$} & \multirow[t]{2}{*}{ Number of Trials } & \multirow[t]{2}{*}{ First Three Trials (s) } & \multirow[t]{2}{*}{ Last Three Trials (s) } & & \\
\hline & & & & & $\mathrm{M}(\mathrm{SE})$ & $t$ \\
\hline \multicolumn{7}{|c|}{ Experiment 1: Common Region to Proximity (Orientation Change) } \\
\hline 3- to 4-month-olds & 20 & $8.75(.75)$ & $31.55(4.09)$ & $11.40(1.71)$ & $46.39(3.74)$ & 0.97 \\
\hline 6- to 7-month-olds & 20 & $10.65(1.25)$ & $21.94(2.44)$ & $9.05(0.91)$ & $56.43(2.28)$ & $2.82 *$ \\
\hline \multicolumn{7}{|c|}{ Experiment 2: Common Region to Proximity (No Orientation Change) } \\
\hline 3- to 4-month-olds & 20 & $8.80(.78)$ & $33.00(3.69)$ & $11.72(1.63)$ & $46.62(4.33)$ & 0.78 \\
\hline \multicolumn{7}{|c|}{ Experiment 3: Proximity to Proximity (No Orientation Change) } \\
\hline 3- to 4-month-olds & 16 & $6.62(.22)$ & $33.17(4.48)$ & $12.24(2.23)$ & $55.91(2.36)$ & $2.51 *$ \\
\hline \multicolumn{7}{|c|}{ Experiment 4: Connectedness to Proximity (Orientation Change) } \\
\hline 3- to 4-month-olds & 20 & $10(1.14)$ & $35.10(4.55)$ & $12.05(1.75)$ & $58.70(3.38)$ & $2.57 *$ \\
\hline
\end{tabular}

$* p<.05$, two-tailed; significantly different from the chance level of $50 \%$

\section{Experiment 2}

The older infants in Experiment 1 exhibited evidence of transfer of learned associations not only by generalizing grouping based on common region to that based on proximity, but also by bridging the vertical/horizontal change in orientation from habituation to test. Prior research with identical stimuli has shown that younger infants are able to generalize grouping across changes in the orientation of common regions (Bhatt et al., 2007; Hayden et al., 2008). Thus, it is unlikely that younger infants' failure to transfer grouping in Experiment 1 was due to the change in orientation from habituation to test. Nevertheless, it is possible that presenting infants during the test with a novel grouping principle in combination with a novel orientation may have increased the complexity of the task beyond younger infants' ability, and this may have led to their failure to generalize. In other words, it is possible that even young infants are capable of generalizing grouping based on one cue (common region) to that based on another (proximity) but this ability is masked in the presence of the additional change in orientation. We examined this issue in Experiment 2 by asking whether younger infants generalize when the orientation change from habituation to test is eliminated, thereby providing a simpler task. Three- to 4-month-olds were habituated to two pairs of elements in different regions, as in Experiment 1. During the novelty preference test, however, the pairs of elements remained in the same orientation as the habituation stimuli.
Method

Participants Twenty 3- to 4-month-olds (11 males; mean age $=103.6$ days, $S D=10.5)$ participated. Data from an additional 10 infants were excluded due to a failure to sample both test stimuli $(n=8)$, fussiness $(n=1)$, or disinterest $(n=1)$. Infants were recruited in the same manner as in Experiment 1.

Stimuli The habituation and test stimuli were the same as those used in Experiment 1 (Fig. 1), except that the orientation of the patterns was not changed between the two phases. For example, if an infant was habituated to regions that were vertically oriented, he/she was tested with proximity-based groupings that were defined by vertical spacing.

Apparatus and procedure The apparatus and procedure were the same as those used in Experiment 1. Coding reliability was assessed by a second coder on a randomly selected subset of 5 infants. The Pearson correlation coefficient between the two coders' scores was .96 during the tests and .93 during habituation.

\section{Results and discussion}

All infants habituated within the 20-trial limit. Their look durations declined over the course of habituation (see Table 1). As was required by the procedure, then, infants were habituated before being tested. As in Experiment 1, 
3- to 4-month-olds failed to exhibit a preference for the novel pairing of elements during test trials, $t(19)=0.78$, $p>.40$ (Table 1). Thus, although the task was simplified in comparison with Experiment 1, young infants still failed to generalize grouping. These results reinforce our finding from Experiment 1 that 3- to 4-month-olds are unable to exhibit transfer of learned associations in the form of generalization from common region to proximity.

\section{Experiment 3}

Young infants' failure to generalize grouping in Experiments 1 and 2 could not have been due to an inability to organize elements on the basis of common region during habituation, as indicated by previous research with infants in this age group with identical stimuli (Bhatt et al., 2007) and even with illusory regions (Hayden et al., 2008). It is possible, however, that young infants' failure to exhibit evidence of generalization may have been due to their failure to group on the basis of proximity during the tests in Experiments 1 and 2. As previously discussed, prior research by Quinn, Bhatt, and Hayden (2008) indicates that young infants can generate global column/row gestalts on the basis of elements' proximity relations. However, the current research involved the segregation of pairs of elements into groups based on proximity. It is possible that the latter task is beyond the capability of young infants. Thus, the younger infants in Experiments 1 and 2 may have failed to exhibit evidence of generalization because they failed to group the four elements seen during the test into two pairs based on proximity.

To examine this issue, infants in the current experiment were tested for their sensitivity to groupings based on proximity during both habituation and testing (Fig. 2). If infants exhibited evidence of grouping under these conditions, then it would indicate that infants are capable of grouping on the basis of proximity even during the two 10-s test trials. In that case, young infants' lack of preference during the tests in Experiments 1 and 2 could not have been due to an inability to organize on the basis of proximity during the test. Rather, their failure was likely due to the lack of transfer from common region to proximity-based grouping.

Method

Participants Sixteen 3- to 4-month-old infants (9 males; mean age $=106.8$ days, $S D=8.4)$ participated in Experiment 3. An additional 3 infants' data were excluded due to sibling interference $(n=1)$, failure to sample both stimuli during test $(n=1)$, or disinterest $(n=1)$.

Stimuli Infants were habituated to proximity-based groupings of elements (Fig. 2). As in Experiments 1

\section{Habituation}

Trial 1

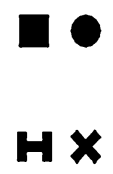

$\square$

$\mathbf{H} \mathbf{X}$

Trial 2

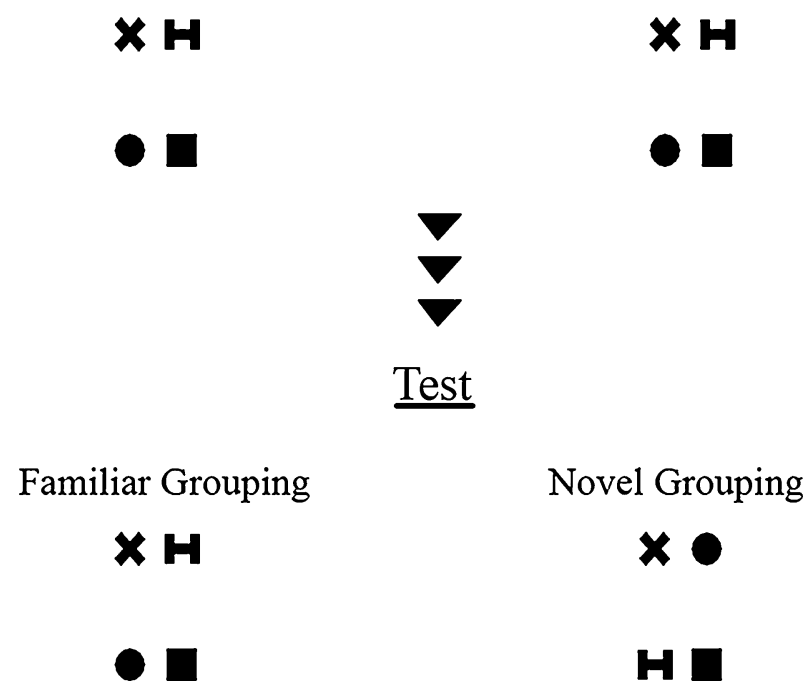

Fig. 2 Examples of the stimuli used in Experiment 3. Pairings during both habituation and test were based on proximity relations. Also, the orientation of the pairs (i.e., whether relations were defined by horizontal or vertical proximity) did not change from habituation to test. Counterbalancing of habituation and novel pairs was the same as in Experiments 1 and 2

and 2, half of the infants were exposed to patterns that led to vertically oriented groupings, while the other half were exposed to horizontally oriented groupings. The stimuli used during the test trials in the present experiment were the same as the test stimuli in Experiments 1 and 2 . During habituation, the specific position of elements varied across trials, although the same two elements always formed a pair, as determined by their proximity to one another. Moreover, as in Experiment 2, there was no change in the vertical/horizontal nature of the grouping from habituation to test.

Apparatus and procedure The same apparatus and procedure as those used in the previous two experiments were also used here. Coding reliability was assessed by a second coder on a randomly selected subset of 5 infants. The Pearson correlation coefficient between the two coders' scores was .97 during the tests and .99 during habituation. 
Results and discussion

All infants habituated within the 20-trial limit. Their look durations declined from the first 3 to the last 3 habituation trials, as required by the habituation procedure (Table 1). During the test trials, infants exhibited a mean novelty preference score that was significantly greater than chance, $t(15)=2.51, p<.05$ (Table 1), thereby indicating that they could group elements on the basis of proximity. Moreover, infants' score in this experiment was greater than the younger infants' score in Experiments 1 and 2, $t(34)=2.03$, $p<.05, d=0.70$, and $t(34)=1.88, p<.05, d=0.61$, respectively. Thus, the young infants' failure to generalize in Experiments 1 and 2 was not due to the inability to group elements on the basis of proximity during the tests.

\section{Experiment 4}

The results of Experiments 1-3 suggest that older infants generalize learned associations under conditions in which younger infants fail. A question that follows is whether this finding reflects a qualitative change in the development of the effects of learning on perceptual organization. That is, is generalization of learned associations unavailable early in life, and does it manifest itself only after 4 months? Clearly, it is logically impossible to prove the absence of a capacity; thus, it is not possible to provide a definitive affirmative answer to the question. On the other hand, evidence of generalization by younger infants under different (presumably, more optimal) conditions would provide a negative answer to the question by indicating that this learning transfer capacity is available even at younger ages, although perhaps not as strong as in older infants. We addressed this possibility in Experiment 4.

Specifically, we examined whether even young infants exhibit evidence of generalization by transferring grouping based on connectedness to grouping based on proximity. We chose connectedness (in the place of the common region cue used in Experiments 1 and 2) because it is considered to a strong organizational cue in adulthood (Palmer \& Rock, 1994) and in infancy (Hayden, Bhatt, \& Quinn, 2006). For example, connectedness is a more potent cue than shape similarity for perceptual organization in infancy (Hayden, Bhatt, \& Quinn, 2009). On the other hand, common region is a cue that differs from many others in the fact that the organizing information (i.e., the regions in which elements are located) is external to the elements themselves (Beck \& Palmer, 2002; Bhatt et al., 2007; Palmer, 1992), and this may make it a relatively weak cue. Thus, it is possible, that the young infants in Experiments 1 and 2 may have failed to transfer grouping based on common region to grouping based on proximity relations because of the relative weakness of the former type of organization. In contrast, grouping based on connectedness may be stronger and provide a better test of young infants' ability to transfer learning.

\section{Method}

Participants Twenty 3- to 4-month-old infants (8 males; mean age $=106.6$ days, $S D=11.08)$ participated in Experiment 4. An additional 7 infants' data were excluded due to fussiness $(n=2)$, failure to sample both stimuli during test $(n=2)$, or disinterest $(n=3)$.

Stimuli The habituation stimuli consisted of pairs of elements grouped on the basis of connectedness; two lines were used to connect the elements (see Fig. 3). The shapes used, the counterbalancing, the vertical/horizontal nature of the organization, and the changing of the positions of the elements across trials were the same as in Experiments 1 and 2. Also, the test stimuli were the same as those used in Experiment 1 (Fig. 3).

\section{Habituation}

Trial 1
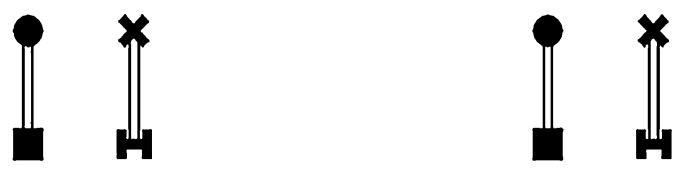

Trial 2
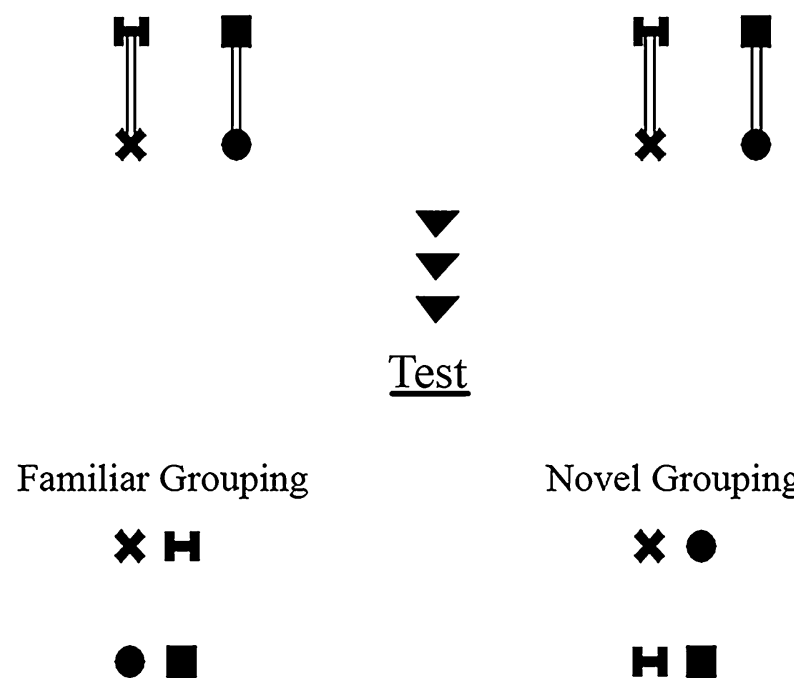

Novel Grouping

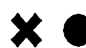

H

Fig. 3 Examples of the stimuli used in Experiment 4. Pairings were based on connectedness and proximity during habituation and test, respectively. In all other respects, the stimuli used were the same as in Experiment 1 
Apparatus and procedure The apparatus and procedure used were the same as those used in the previous experiments. Coding reliability was assessed by a second coder on a randomly selected subset of 5 infants. The Pearson correlation coefficient between the two coders' scores was .97 during the tests and .86 during habituation.

\section{Results and discussion}

One infant failed to habituate within the 20-trial limit. The pattern of results was the same whether or not this infant's data were included. In the following analyses, all the data are included. Infants' look durations (Table 1) decreased from the first 3 to the last 3 habituation trials, $t(19)=6.75$, $p<.001$, as required by the procedure. This indicates that infants were habituated to the criterion level before being tested. During the test trials, infants exhibited a mean novelty preference score that was significantly greater than the chance level of $50 \%, t(19)=2.57, p<.05$. Thus, 3 - to 4-month-old infants exhibited evidence of generalization by transferring grouping from connectedness to proximity. Moreover, the infants' score in this experiment was significantly greater than the younger infants' scores in Experiment 1 and Experiment 2, t(38) $=2.44, p<.05, d=$ 0.77 , and $t(38)=2.20, p<.05, d=0.69$, respectively. Therefore, in contrast to the findings of Experiments 1 and 2 , the use of connectedness as the initial grouping cue in Experiment 4 led even young infants to transfer learned associations to a novel context.

\section{General discussion}

Transfer of learned associations is a critical psychological function. It allows us to process stimuli around us at the appropriate level of grouping even in novel situations. For instance, when reading, it is more effective to function at the level of words than at the level of individual letters, and expert readers exhibit this capacity even when reading novel texts (Goldstone, 1998). This is accomplished by transferring learned letter associations to novel situations. In the present experiments, 6- to 7-month-olds and 3- to 4month-olds generalized grouping based on one organizational cue to another, indicating that they had combined the elements in one context and carried forward the combinations to a new context. These results suggest that previously learned associations can impact future perceptual functioning in infancy, as shown in the case of adults by Vickery and Jiang (2009).

The fact that generalization of learned grouping was exhibited by young infants indicates that, as in the case of many other aspects of cognitive development (for reviews, see Bhatt \& Quinn, 2011; Bremner, 2007; Johnson, 2010; Kellman \& Arterberry, 1998; Woodward \& Needham, 2009), functional perceptual learning mechanisms are evident early in life. This early onset is consistent with the emergence of other forms of learning, such as classical and operant conditioning and imitation, that have been shown to be functional soon after birth (e.g., Blass, Ganchrow, \& Steiner, 1984; Meltzoff \& Moore, 1983; Rovee \& Rovee, 1969).

The present findings are also consistent with the proposal by Bhatt and Quinn (2011) that perceptual learning is qualitatively similar across ages. Bhatt and Quinn proposed that, although older infants may learn faster and better in some conditions, there is unlikely to be complete absence at young ages of some capacity such as generalization of learning. Consistent with this proposal, younger infants generalized learned associations in Experiment 4.

However, a developmental change was documented in the present experiments. Older infants generalized grouping based on common region to grouping based on proximity in Experiment 1, but younger infants failed to do so. This developmental change is consistent with Cohen's (2010) model of perceptual development. This model assumes that older infants tend to function at a more global perceptual level than younger infants. This proposal is based on findings that sensitivity to correlations among features improves with age in infancy (Younger \& Cohen, 1986). Comparable kinds of developmental changes in the processing of relational information have been documented at different ages and in other domains. For instance, Gentner and her colleagues have extensively documented developmental changes in childhood in the processing of similarity relations (e.g., Gentner, Ratterman, Markman, \& Kotovsky, 1995). Similarly, Halford et al. (1995) argued that increases in processing capacity during development in childhood lead to changes in performance on tasks that involve relational information processing, such as analogical reasoning. Developmental changes in the processing of configural and holistic relational aspects of facial features have also been reported in infancy (e.g., Bhatt et al., 2005; Cashon \& Cohen, 2004) and from childhood to adulthood (e.g., Carey \& Diamond, 1994; Diamond \& Carey, 1986; Mondloch \& Thomson, 2008).

It should be noted, however, that the developmental change that was obtained in the present study was not based on a failure to process correlations. Three- to 4-month-olds in Bhatt et al. (2007) exhibited sensitivity to correlations among elements on the basis of common region with the identical stimuli used in the present study; also, infants in Experiment 3 exhibited grouping based on proximity. Rather, young infants failed to transfer grouping between common region and proximity. 
Although infants' failure to generalize learning in Experiments 1 and 2 may not have been due to a failure to process correlations, their performance may have been affected by the strength of the correlation. Specifically, the contrast between young infants' failure to transfer grouping from common region to proximity (Experiments 1 and 2) versus their successful transfer from connectedness to proximity (Experiment 4) might originate from association formation based on common region being weaker than that based on connectedness. As was discussed earlier, connectedness is considered to be a fundamental cue that generates the basic building blocks of images that are then organized using other cues (Hayden et al., 2006, 2009; Palmer \& Rock, 1994), whereas common region is a cue that is external to the elements themselves and, on this basis, might be a weaker cue. Thus, although young infants can group on the basis of common region and proximity, their grouping may not be strong enough to allow transfer from one context to the other. Models of neural development suggest that communication between the dorsal and ventral processing streams (the "where" and "what" streams, respectively) is weak early in life (e.g., Leslie, $\mathrm{Xu}$, Tremoulet, \& Scholl, 1998; Mareschal \& Johnson, 2003). It is therefore possible that the communication between the dorsal (spatial location) and ventral (element shape) processing streams was not very effective in young infants, and this may have led to weak grouping based on common region. By this account, however, the communication between the two streams was sufficiently effective even in young infants to support strong enough grouping based on connectedness for them to transfer to proximity-based relations in Experiment 4.

An additional factor that may have led to differences in young infants' performance on the common region to proximity (Experiments 1 and 2) versus connectedness to proximity tasks (Experiment 4) may be the degree of contextual change. The change from common region to proximity could have been a greater alteration than the one from connectedness to proximity, leading to poorer transfer in the former condition. On this view, older infants' performance in Experiment 1 did not similarly suffer due to the contextual alteration from common region to proximity, suggesting a developmental change in the degree of tolerance to contextual differences. Such a possibility is supported by parallel findings in the literature: For instance, in memory tasks, younger infants are affected more by contextual changes than are older infants (e.g., Hayne, Boniface, \& Barr, 2000; Herbert \& Hayne, 2000). Thus, the strength of grouping and/or the degree of contextual change may have contributed to the developmental change in generalization documented in Experiment 1 and to the differences in young infants' performance in Experiments 1 and 2 versus Experiment 4.
Another point worth noting is the fact that generalization of the sort demonstrated in the present experiments is necessary for a powerful form of perceptual learningnamely, unitization (Goldstone, 1998). Unitization refers to the grouping of lower-level elements into higher-level units that then function as independent features (Pomerantz, 2003, 2006). It leads to the efficient processing of information by enabling the functionally most useful level of analysis. For instance, Shiffrin and Lightfoot (1997) found that repeated exposure to line segment features led adults to combine them into units, which then enabled them to rapidly detect conjunctions of features in a visual search task. Similarly, expert readers unitize letters into words (Goldstone, 1998) because the efficiency of reading is increased by responding to words as units, rather than at the level of individual letters. Also, combining components of objects into higher-order units facilitates object recognition (Goldstone, 2003). To demonstrate unitization, it is necessary to show that learned associations function as units in new situations, similar to the generalization demonstrated in the present experiments. However, in some instances (e.g., Shiffrin \& Lightfoot, 1997), unitization has been shown by demonstrating that the learned associations function as fundamental features (e.g., they "pop out" in visual search situations) and/or lead to configural superiority effects (i.e., superior detection of a configuration, as compared with the features that make up the configuration; see Pomerantz, 2003, 2006). Thus, in future studies, it will be important to determine whether learned associations of the sort shown in the present experiments result in pop-out and configural superiority effects in infancy.

It is also worth noting that the learning exhibited in the present experiments in the form of transfer of grouping may be an example of biological and learning processes working synergistically. If the tendencies early in life to organize using cues such as common region and connectedness are inherently "built into" the perceptual system, the learning that occurs (i.e., the association of the elements) as a result of the functionality of the grouping principles can be construed as having been bootstrapped onto those principles. This observation is consistent with the broader idea that both the development of perceptual organization and its functioning in adulthood are adaptive outcomes of the interplay between core capacities originating from our biological endowment and knowledge acquired via experience (Baillargeon, Li, Ng, \& Yuan, 2009; Bhatt \& Quinn, 2011; Johnson, 2009).

Author Note This research was supported by NIH Grants HD-52724, MH-086958, and HD-46526. We thank the infants and the parents who participated in this study. Address correspondence to Ramesh S. Bhatt, Department of Psychology, University of Kentucky, Lexington, KY 40506-0044. Email: rbhatt@email.uky.edu. 


\section{References}

Baillargeon, R., Li, J., Ng, W., \& Yuan, S. (2009). An account of infants' physical reasoning. In A. Needham \& A. Woodward (Eds.), Learning and the infant mind (pp. 66-116). New York: Oxford University Press.

Beck, D. M., \& Palmer, S. E. (2002). Top-down influences on perceptual grouping. Journal of Experimental Psychology. Human Perception and Performance, 28, 1071-1084.

Bhatt, R. S., Bertin, E., Hayden, A., \& Reed, A. (2005). Face processing in infancy: Developmental changes in the use of different kinds of relational information. Child Development, 76, 169-181.

Bhatt, R. S., Hayden, A., \& Quinn, P. C. (2007). Perceptual organization based on common region in infancy. Infancy, 12, 147-168.

Bhatt, R. S., Hayden, A., Reed, A., Bertin, E., \& Joseph, J. E. (2006). Infants' perception of information along object boundaries: Concavities versus convexities. Journal of Experimental Child Psychology, 94, 91-113.

Bhatt, R. S., \& Quinn, P. C. (2011). How does learning impact development in infancy? The case of perceptual organization. Infancy, 16, 2-38.

Bhatt, R. S., \& Rovee-Collier, C. (1994). Perception and 24-hour retention of feature relations in infancy. Developmental Psychology, $30,142-150$.

Bhatt, R. S., \& Rovee-Collier, C. (1996). Infant's forgetting of correlated attributes and object recognition. Child Development, 67, 172-187.

Blass, E. M., Ganchrow, J. R., \& Steiner, J. E. (1984). Classical conditioning in newborn humans, 2-48 hours of age. Infant Behavior \& Development, 7, 223-235.

Bremner, J. G. (2007). Perception and knowledge of the world. In A. Slater \& M. Lewis (Eds.), Introduction to infant development (2nd ed., pp. 137-152). New York: Oxford University Press.

Carey, S., \& Diamond, R. (1994). Are faces perceived as configurations more by adults than by children? Visual Cognition, 1, 253-274.

Cashon, C. H., \& Cohen, L. B. (2004). Beyond U-shaped development in infants' processing of faces: An information-processing account. Journal of Cognition and Development, 5, 59-80.

Cohen, L. B. (1991). Infant attention: An information processing approach. In M. J. Weiss \& P. R. Zelazo (Eds.), Newborn attention: Biological constraints and the influence of experience (pp. 1-21). Norwood: Ablex.

Cohen, L. B. (2004). Uses and misuses of habituation and related preference paradigms. Infant and Child Development, 13, 349352.

Cohen, L. B. (2010). A bottom-up approach to infant perception and cognition: A summary of evidence and discussion of issues. In $\mathrm{S}$. P. Johnson (Ed.), Neoconstructivism: The new science of cognitive development (pp. 335-346). New York: Oxford University Press.

Diamond, R., \& Carey, S. (1986). Why faces are and are not special: An effect of expertise. Journal of Experimental Psychology. General, 115, 107-117.

Feigenson, L., \& Halberda, J. (2008). Conceptual knowledge increases infants' memory capacity. Proceedings of the National Academy of Sciences, 105, 9926-9930.

Fiser, J., \& Aslin, R. N. (2002). Statistical learning of new visual feature combinations by infants. Proceedings of the National Academy of Sciences, 99, 15822-15826.

Gentner, D., Ratterman, M. J., Markman, A., \& Kotovsky, L. (1995). Two forces in the development of relational similarity. In T. J. Simon \& G. S. Halford (Eds.), Developing cognitive competence: New approaches to process modeling (pp. 263-313). Hillsdale: Erlbaum.
Gibson, E. J. (1969). Principles of perceptual learning and development. New York: Meredith Corporation.

Goldstone, R. L. (1998). Perceptual learning. Annual Review of Psychology, 49, 585-612.

Goldstone, R. L. (2003). Learning to perceive while perceiving to learn. In R. Kimchi, M. Behrmann, \& C. R. Olson (Eds.), Perceptual organization in vision: Behavioral and neural perspectives (pp. 223-278). Mahwah: Erlbaum.

Halford, G. S., Smith, S. B., Dickson, J. C., Mayberry, M. T., Kelly, M. E., Bain, J. D., et al. (1995). Modeling the development of reasoning strategies: The role of analogy, knowledge, and capacity. In T. J. Simon \& G. S. Halford (Eds.), Developing cognitive competence: New approaches to process modeling (pp. 77-156). Hillsdale: Erlbaum.

Hayden, A., Bhatt, R. S., \& Quinn, P. C. (2006). Infants's sensitivity to uniform connectedness as a cue for perceptual organization. Psychonomic Bulletin \& Review, 13, 257-261.

Hayden, A., Bhatt, R. S., \& Quinn, P. C. (2008). Perceptual organization based on illusory regions in infancy. Psychonomic Bulletin \& Review, 15, 443-447.

Hayden, A., Bhatt, R. S., \& Quinn, P. C. (2009). Relations between uniform connectedness, luminance, and shape similarity as perceptual organizational cues in infancy. Attention, Perception, \& Psychophysics, 71, 52-63.

Hayne, H., Boniface, J., \& Barr, R. (2000). The development of declarative memory in human infants: Age-related changes in deferred imitation. Behavioral Neuroscience, 114, 77-83.

Herbert, J., \& Hayne, H. (2000). Memory retrieval by 18-30-month-olds: Age-related changes in representational flexibility. Developmental Psychology, 36, 473-484.

Horowitz, F. D., Paden, L., Bhana, K., \& Self, P. (1972). An infant-control procedure for studying infant visual fixation. Developmental Psychology, 7, 90.

Hunter, M. A., \& Ames, E. W. (1988). A multifactor model of infant preferences for novel and familiar stimuli. In L. P. Lipsitt (Ed.), Advances in child development and behavior (pp. 69-95). New York: Academic Press.

Johnson, S. P. (2009). The role of learning in cognitive development: Challenges and prospects. In A. Needham \& A. Woodward (Eds.), Learning and the infant mind (pp. 286-295). New York: Oxford University Press.

Johnson, S. P. (2010). Perceptual completion in infancy. In S. P. Johnson (Ed.), Neoconstructivism: The new science of cognitive development (pp. 47-65). New York: Oxford University Press.

Kellman, P. J., \& Arterberry, M. E. (1998). The cradle of knowledge: Development of perception in infancy. Cambridge: MIT press.

Kirkham, N. Z., Slemmer, J. A., \& Johnson, S. P. (2002). Visual statistical learning in infancy: Evidence for a domain general learning mechanism. Cognition, 83, B35-B42.

Koffka, K. (1935). Principles of Gestalt psychology. New York: Harcourt, Brace \& World.

Kohler, W. (1929). Gestalt psychology. New York: Horace Liveright.

Leslie, A. M., Xu, F., Tremoulet, P. D., \& Scholl, B. J. (1998). Indexing and the object concept: Developing "what" and "where" systems. Trends in Cognitive Sciences, 2, 10-18.

Mareschal, D., \& Johnson, M. H. (2003). The "what" and "where" of object representations in infancy. Cognition, 88, 259-276.

Meltzoff, A. N., \& Moore, M. K. (1983). Newborn infants imitate adult facial gestures. Child Development, 54, 702-709.

Mondloch, C. J., \& Thomson, K. (2008). Limitation in four-year-old children's sensitivity to the spacing among facial features. Child Development, 79, 1513-1523.

Murphy, G. L. (2002). The big book of concepts. Cambridge: MIT Press.

Palmer, S. E. (1992). Common region: A new principle of perceptual grouping. Cognitive Psychology, 24, 436-447. 
Palmer, S. E. (1999). Vision science: Photons to phenomenology. Cambridge: MIT Press.

Palmer, S. E., \& Rock, I. (1994). Rethinking perceptual organization: The role of uniform connectedness. Psychonomic Bulletin \& Review, 1, 29-55.

Peterson, M. A. (1994). Shape recognition can and does occur before figure-ground organization. Current Directions in Psychological Science, 3, 105-111.

Peterson, M. A., \& Salvagio, E. (2008). Inhibitory competition in figure-ground perception: Context and convexity. Journal of Vision, 8, 1-13.

Pomerantz, J. R. (2003). Wholes, holes, and basic features in vision. Trends in Cognitive Sciences, 7, 471-473.

Pomerantz, J. R. (2006). Color as a Gestalt: Pop out with basic features and with conjunctions. Visual Cognition, 14, 619-628.

Quinn, P. C., Bhatt, R. S., Brush, D., Grimes, A., \& Sharpnack, H. (2002). Development of form similarity as a Gestalt grouping principle in infancy. Psychological Science, 13, 320-328.

Quinn, P. C., Bhatt, R. S., \& Hayden, A. (2008). Young infants readily use proximity to organize visual pattern information. Acta Psychologica, 127, 289-298.

Roder, B. J., Bushnell, E. W., \& Sasseville, A. M. (2000). Infants' preference for familiarity and novelty during the course of visual processing. Infancy, 1, 491-507.
Rovee, C. K., \& Rovee, D. T. (1969). Conjugate reinforcement of infant exploratory behavior. Journal of Experimental Child Psychology, 8, 33-69.

Saffran, J. S. (2009). What can statistical learning tell us about infant learning? In A. Needham \& A. Woodward (Eds.), Learning and the infant mind (pp. 29-46). New York: Oxford University Press.

Shiffrin, R. M., \& Lightfoot, N. (1997). Perceptual learning of alphanumeric-like characters. In R. L. Goldstone, P. G. Schyns, \& D. L. Medin (Eds.), The psychology of learning and motivation, vol. 36 (pp. 45-82). San Diego: Academic Press.

Slater, A., Mattock, A., Brown, E., Burnham, D., \& Young, A. (1991). Visual processing of stimulus compounds in newborn infants. Perception, 20, 29-33.

Vickery, T. J., \& Jiang, Y. V. (2009). Associative grouping: Perceptual grouping of shapes by association. Attention, Perception, \& Psychophysics, 71, 896-909.

Wertheimer, M. (1958). Principles of perceptual organization. In D. C. Beardslee \& M. Wertheimer (Eds.), Readings in perception (pp. 115135). Princeton: Van Nostrand. Original work published 1923.

Woodward, A. L., \& Needham, A. (Eds.). (2009). Learning and the infant mind. New York: Oxford University Press.

Younger, B. A., \& Cohen, L. B. (1986). Developmental change infants' perception of correlations among attributes. Child Development, 57, 803-815. 